\title{
CAST LISTS
}

\section{The New Theatre (now the Noël Coward Theatre), London, 1936}

Director: Theodore Komisarjevsky

Designer: Theodore Komisarjevsky

\author{
Cleopatra \\ Antony \\ Enobarbus \\ Charmian \\ Iras \\ Alexas \\ Other parts played by: \\ Lawrence Anderson \\ George Hayes \\ Ellis Irving \\ Vernon Kelso \\ Ion Swinley \\ Arthur Young
}

Eugenie Leontovich

Donald Wolfit

Leon Quartermaine

Margaret Rawlings

Vera Poliakoff

Hubert Harben

\section{Shakespeare Memorial Theatre, Stratford-upon-Avon, 1953}

Director: Glen Byam Shaw

Designer: Motley (Margaret Harris)

\author{
Cleopatra \\ Antony \\ Octavius Caesar \\ Lepidus, Diomedes \\ Domitius Enobarbus \\ Octavia \\ Sextus Pompeius \\ Charmian \\ Iras
}

Slave

Mardian

Agrippa
Peggy Ashcroft

Michael Redgrave

Marius Goring

Donald Pleasence

Harry Andrews

Rachel Kempson

Tony Britton

Jean Wilson

Mary Watson

Anthony Adams

Mervyn Blake

John Bushelle 


$\begin{array}{ll}\text { Nubian Slave Girl } & \text { Diana Chadwick } \\ \text { Dancing Girl } & \text { Marigold Charlesworth } \\ \text { Sailor, Soldier, Slave } & \text { Dennis Clinton } \\ \text { Slave } & \text { James Culliford } \\ \text { Soldier } & \text { Nigel Davenport } \\ \text { Dercetas, Sailor, Slave } & \text { Peter Duguid } \\ \text { Maecenas } & \text { Donald Eccles } \\ \text { Slave } & \text { John Glendenning } \\ \text { Gallus, Sailor, Slave } & \text { Denys Graham } \\ \text { Silius, Slave } & \text { Charles Gray } \\ \text { Old Soldier, Slave } & \text { George Hart } \\ \text { Ventidius, Soldier } & \text { Michael Hayes } \\ \text { Soldier } & \text { Charles Howard } \\ \text { Soldier } & \text { Peter Johnson } \\ \text { Seleucus, Soldier } & \text { Gareth Jones } \\ \text { Demetrius, Taurus, Soldier } & \text { Bernard Kay } \\ \text { Slave } & \text { David King } \\ \text { Slave } & \text { Cavan Malone } \\ \text { Soldier, Slave } & \text { Richard Martin } \\ \text { Canidius, Soothsayer, Soldier } & \text { Philip Morant } \\ \text { Sicyon, Messenger, Euphronius } & \text { Peter Norris } \\ \text { Eros, Slave } & \text { David O'Brien } \\ \text { Thidias } & \text { William Peacock } \\ \text { Soldier } & \text { John Roberts } \\ \text { Cabin Boy } & \text { Robert Scroggins } \\ \text { Dolabella } & \text { Robert Shaw } \\ \text { Slave } & \text { Raymond Sherry } \\ \text { Proculeius, Messenger } & \text { Powys Thomas } \\ \text { Alexas, Soldier } & \text { Alan Townsend } \\ \text { Philo, Sailor } & \text { Michael Turner } \\ \text { Menas } & \text { Michael Warre } \\ \text { Clown, Slave, Soldier } & \text { James Wellman } \\ \text { Scarus, Slave } & \text { Jerome Willis } \\ & \end{array}$

\section{Citizens' Theatre, Glasgow, 1972}

Director: Giles Havergal Designer: Philip Prowse

Cleopatra

Antony

Octavius Caesar

Acolyte

Acolyte
Jonathan Kent John Duttine

Mike Gwilym

Cheryl Campbell Angela Chadfield 


\title{
Royal Shakespeare Theatre, Stratford-upon-Avon, 1972
}

\author{
Directors: Trevor Nunn, Buzz Goodbody \\ Set designer: Christopher Morley \\ Costume designer: Ann Curtis
}

Cleopatra
Mark Antony
Octavius Caesar
Lepidus
Domitius Enobarbus
Octavia
Sextus Pompeius
Charmian
Iras

Alexas

Canidius, King

Waiting Woman

Demetrius

Lamprius

Diomedes, Pacorus

Messenger

Gallus

Ventidius

King, Soldier 5, Varrius

Eunuch

Waiting Woman

Eunuch, Galley Servant 2

Maecenas

Guard 1, Servant

Scarus

Menas

Decretas

Clown

Silius

King, Silvius, Soldier 6

Mardian

Thidias

Eros

Agrippa

Dolabella
Janet Suzman

Richard Johnson

Corin Redgrave

Raymond Westwell

Patrick Stewart

Judy Cornwell

Gerald James

Rosemary McHale

Mavis Taylor Blake

Darien Angadi

John Atkinson

Wendy Bailey

John Bardon

John Bott

Loftus Burton

Joseph Charles

Thomas Chesleigh

Constantin De Goguel

Hans De Vries

Michael Egan

Edwina Ford

Paul Gaymon

Patrick Godfrey

Peter Godfrey

Don Henderson

Ian Hogg

Jonathan Holt

Geoffrey Hutchings

Christopher Jenkinson

Malcolm Kaye

Sidney Livingstone

Calvin Lockhart

Joe Marcel

Clement McCallin

Martin Milman 
King, Menecrates, Soldier 1

Servant 1

Euphronius

Proculeius

Servant, Soldier 3

Eunuch, Seleucus

Guard 2, Servant

Servant, Soldier 2

King, Taurus

Messenger, Sentry

Galley Servant 1, King, Soldier 4
Robert Oates

Tony Osoba

Lennard Pearce

Timothy Pigott-Smith

Michael Radcliffe

Jason Rose

Simon Rouse

Kevan Sheehan

Desmond Stokes

Keith Taylor

Arthur Whybrow

\section{Royal Shakespeare Theatre, Stratford-upon-Avon, 1978}

Director: Peter Brook

Designer: Sally Jacobs

Cleopatra

Mark Antony

Octavius Caesar

Lepidus

Enobarbus

Octavia

Pompey

Charmian

Iras

Unnamed parts

Philo, Scarus

Proculeius, Soothsayer, unnamed parts

Canidius, Gallus, unnamed parts

Decretas, Taurus, Varrius, unnamed parts

Clown, Messenger

Demetrius, Maecenas

Mardian, Schoolmaster, unnamed parts

Boy Singer, Eros

Menas

Dolabella, Ventidius

Menecrates, Seleucus, unnamed parts

Alexas, Thidias, unnamed parts
Glenda Jackson

Alan Howard

Jonathan Pryce

Paul Brooke

Patrick Stewart

Marjorie Bland

David Suchet

Paola Dionisotti

Juliet Stevenson

Alan Barker

John Bowe

David Bradley

Dennis Clinton

Alan Cody

Richard Griffiths

David Lyon

Philip McGough

Hilton McRae

Paul Moriarty

John Nettles

George Raistrick

Alan Rickman 
Agrippa

Old Soldier

Diomedes, Silius, unnamed parts
Paul Webster

Raymond Westwell

Paul Whitworth

\section{The Other Place, Stratford-upon-Avon, 1982}

Director: Adrian Noble

Designer: Nadine Baylis

Cleopatra

Mark Antony

Octavius Caesar

Lepidus, Old Soldier, Proculeius

Domitius Enobarbus

Octavia

Sextus Pompeius, Dolabella

Charmian

Iras

Thidias, Alexas

Mardian

Maecenas, Euphronius

Canidius, Seleucus

Eros

Decretas, Menecrates

Taurus, Menas

Agrippa

Clown

Varrius, Diomedes
Helen Mirren

Michael Gambon

Jonathan Hyde

Paul Webster

Bob Peck

Penelope Beaumont

Clive Wood

Sorcha Cusack

Josette Simon

Ken Bones

Michael Fitzgerald

David Glover

Nigel Harrison

Michael Maloney

Tom Mannion

Niall Padden

George Parsons

David Troughton

Albie Woodington

Olivier Theatre, National Theatre, London, 1987

Director: Peter Hall

Designer: Alison Chitty

Cleopatra

Mark Antony

Octavius

Lepidus, Clown

Domitius Enobarbus

Octavia

Sextus Pompey

Charmian

Iras
Judi Dench

Anthony Hopkins

Tim Pigott-Smith

John Bluthal

Michael Bryant

Sally Dexter

David Schofield

Miranda Foster

Helen Fitzgerald 
Silius, Thidias, Gallus

Scarus, Alexas, Diomedes

Soldier

Dolabella, Varrius

Soldier

Decretas, Menas

Eros

Menecrates, Schoolmaster

Philo

Agrippa

Soldier

Soldier

Mardian

Lady attending on Octavia

Egyptian, Soldier

Maecenas

Demetrius, Proculeius, Ventidius

Canidius, Seleucus, Soothsayer

Boy
Desmond Adams

Robert Arnold

Ian Bolt

Michael Bottle

Patrick Brennan

Michael Carter

Jeremy Flynn

Peter Gordon

Mike Hayward

Basil Henson

Hus Levent

Simon Needs

Iain Ormsby-Knox

Frances Quinn

Simon Scott

Graham Sinclair

Brian Spink

Daniel Thorndike

Peter Corey, Paul Vinhas

\section{Shakespeare Theatre Company, Folger Shakespeare Library, Washington DC, 1988}

Director: Michael Kahn

Set designer: Robert Edward Darling

Costume designer: Judith Dolan

Cleopatra

Antony

Octavius Caesar

Aemilius Lepidus, Schoolmaster

Domitius Enobarbus

Octavia

Sextus Pompeius, Dolabella

Charmian

Iras

Messenger

Ensemble

A Rural Fellow, A Soothsayer

Thidias, Menas, Ensemble

Mardian

Ensemble

Ensemble

Ensemble
Franchelle Stewart Dorn

Kenneth Haigh

Michel R. Gill

Emery Battis

Jack Ryland

Katrina Van Duyn

Edward Gero

Gail Grate

Leah Maddrie

Oliver Barreiro

Mark Douglas

Charles Dumas

Michael Forrest

Carlos Juan Gonzalez

Steve Harley

Marvin E. Hart

James Huesz 
Alexas

Attendant to Octavia

Agrippa

Ensemble

Canidius, Proculeius

Demetrius, Ensemble

Maecenas

Scarus

Eros

Attendant to Octavia

Menecrates, Ensemble
Robert Jason

Linda Khoury

Floyd King

Mykal Knight

Barry Mulholland

Jan Notzon

K. Lype O’Dell

Paris Peet

Andrew Land Prosky

Lisa Rhoden

D. Raymond Simonton

\title{
Wiener Festwochen and the Berliner Ensemble (co-production), King's Theatre (Edinburgh Festival), 1994
}

\author{
Director: Peter Zadek \\ Set designer: Wilfried Minks \\ Costume designer: Norma Moriceau \\ German translation: Elisabeth Plessen
}

\begin{tabular}{ll} 
Cleopatra & Eva Mattes \\
Antony & Gert Voss \\
Octavius & Veit Schubert \\
Lepidus & Jaecki Schwarz \\
Enobarbus & Hermann Beyer \\
Octavia, Iras & Gaby Herz \\
Pompey, Taurus, Diomedes & Georg Bonn \\
Charmian & Deborah Kaufmann \\
& \\
Eros & Uwe Bohm \\
Scarus, Messenger to Cleopatra & Hans Fleischmann \\
Menas, Schoolmaster, Soothsayer, & Urs Hefti \\
\multicolumn{1}{c}{ Clown } & \\
Agrippa & Dieter Knaup \\
Proculeius, Thidias & Rüdiger Kuhlbrodt \\
Varrius, Egyptian Soldier & Patrick Lanagan \\
Canidius, Seleucus & Stefan Lisewski \\
Servant to Cleopatra & Christoph Müller \\
Old Soldier & Hans-Peter Reinecke \\
Servant & Lothar Runkel \\
Mardian & Nino Sandow \\
Maecenas & Martin Seifert \\
Dolabella, Alexas & Götz Schulte
\end{tabular}


Demetrius, Gallus

Servant to Cleopatra

Ventidius, Menecrates
Thomas Sicker

Thomas Wendrich

Axel Werner

\section{Northern Broadsides, Halifax, Yorkshire, and UK tour, 1995}

Director: Barrie Rutter

Designer: Jessica Worrall

Cleopatra

Antony

Caesar

Lepidus, Thidias

Enobarbus

Iras, Octavia

Pompey, Eros, Dolabella

Charmian

Agrippa

'Antony', Maecenas

Soothsayer

Menas, Proculeius

Mardian

Messenger

'Cleopatra', Ventidius
Ishia Bennison

Barrie Rutter

Andrew Cryer

Roy North

Dave Hill

Deborah McAndrew

John Gully

Julie Livesey

Stephen Anderson

David Fenwick

David Findlay

Gerard McDermott

David Peacock

Andy Wear

Andrew Whitehead

\section{The Globe Theatre, London, 1999}

Master of play and verse: Giles Block Master of clothing and properties: Jenny Tiramani

Cleopatra

Mark Antony

Octavius Caesar

Lepidus, Philo, A Schoolmaster,

Proculeius

Enobarbus, Seleucus

Octavia, Thidias, Varrius

Sextus Pompeius, Taurus,

Diomedes

Charmian

Iras, The Boy
Mark Rylance

Paul Shelley

Ben Walden

Timothy Davies

John McEnery

Toby Cockerell

Mark Lewis Jones

Danny Sapani

James Gillan 
Maecenas

Decretas, Soothsayer, Cleopatra's

Messenger

Silius, Eros, Gallus, Menecrates

Demetrius, Agrippa

Dolabella, Mardian

Ventidius, Canidius, Alexas, Clown

Scarus, Menas
Jimmy Gardner

Roger Gartland

Liam Hourican

Terence Maynard

Quill Roberts

Michael Rudko

Benedict Wong

\section{Royal Exchange Theatre, Manchester, 2005}

Director: Braham Murray

Designer: Johanna Bryant

Cleopatra
Antony
Caesar
Lepidus, Thidias, Watch
Enobarbus
Octavia, Iras
Charmian

Scarus

Alexas, Seleucus

Agrippa

Maecenas, Schoolmaster, Diomedes

Philo, Clown

Dercetus

Mardian

Dolabella, Sentry

Soothsayer, Eros
Josette Bushell-Mingo

Tom Mannion

Steven Robertson

Will Tacey

Terence Wilton

Gugu Mbatha-Raw

Sarah Paul

Glenn Chapman

Chris Hannon

James Howard

Jack Lord

Joseph Mawle

Fergus O'Donnell

Ali Sichilongo

Simeon Truby

Everal A. Walsh

\section{Royal Shakespeare Company, The Public and GableStage (co-production), Swan Theatre, Stratford-upon-Avon, 2013}

Director: Tarell Alvin McCraney

Designer: Tom Piper

Cleopatra

Mark Antony

Octavius Caesar

Lepidus, Proculeius

Enobarbus

Octavia, Iras
Joaquina Kalukango

Jonathan Cake

Samuel Collings

Henry Stram

Chukwudi Iwuji

Charise Castro Smith 
Pompey, Alexas, Scarus

Charmian, Menas

Agrippa, Thyreus

Mardian, Soothsayer, Eros
Ash Hunter

Sarah Niles

Ian Lassiter

Chivas Michael

\section{Tom Patterson Theatre, Stratford Festival of Canada, Ontario, 2014}

Director: Gary Griffin

Designer: Charlotte Dean

\author{
Cleopatra \\ Mark Antony \\ Octavius Caesar \\ Lepidus \\ Enobarbus \\ Octavia \\ Pompey, Clown \\ Charmian \\ Iras
}

Mecenas

Eros

Man from Sicyon

Agrippa

Diomedes

Towrus

Dolabella, Thidias

Varrius

Scarrus

Ventidius

Menas, Camidius

Soothsayer

Alexas, Proculeius

Mardian

\author{
Yanna McIntosh \\ Geraint Wyn Davies \\ Ben Carlson \\ Randy Hughson \\ Tom McCamus \\ Carmen Grant \\ Brian Tree \\ Sophia Walker \\ Jennifer Mogbock \\ Sean Arbuckle \\ Daniel Briere \\ Ryan Field \\ Peter Hutt \\ Andrew Lawrie \\ Jamie Mac \\ Anthony Malarky \\ André Morin \\ Karack Osborn \\ Andrew Robinson \\ Brad Rudy \\ Stephen Russell \\ E. B. Smith \\ Antoine Yared
}

\section{Toneelgroep Amsterdam, Theater Carré, 2018}

Director: Ivo van Hove

Set (and lighting) designer: Jan Versweyveld

Costume designer: Lies van Assche

Cleopatra

Antonius

Octavius Caesar
Chris Nietvelt

Hans Kesting

Maria Kraakman 
Lepidus

Enobarbus

Octavia

Charmian

Iras

Agrippa

Diomedes

Proculeius

Dolabella

Thidias

Ventidius
Fred Goessens

Bart Slegers

Hélène Devos

Marieke Heebink

Frieda Pittoors

Gijs Scholten van Aschat

Janni Goslinga

Hugo Koolschijn

Alwin Pulinckx

Harm Duco Schut

Eelco Smits 